\title{
IDENTIFICATION OF LINEAR AND NONLINEAR AERODYNAMIC IMPULSE RESPONSES USING DIGITAL FILTER TECHNIQUES
}

\author{
Walter A. Silva* \\ Aeroelasticity Branch \\ NASA Langley Research Center
}

\begin{abstract}
This paper discusses the mathematical existence and the numerically-correct identification of linear and nonlinear aerodynamic impulse response functions. Differences between continuous-time and discrete-time system theories, which permit the identification and efficient use of these functions, will be detailed. Important input/output definitions and the concept of linear and nonlinear systems with memory will also be discussed. It will be shown that indicial (step or steady) responses (such as Wagner's function), forced harmonic responses (such as Theodorsen's function or those from doublet lattice theory), and responses to random inputs (such as gusts) can all be obtained from an aerodynamic impulse response function. This paper establishes the aerodynamic impulse response function as the most fundamental, and, therefore, the most computationally efficient, aerodynamic function that can be extracted from any given discrete-time, aerodynamic system. The results presented in this paper help to unify the understanding of classical two-dimensional continuous-time theories with modern threedimensional, discrete-time theories. First, the method is applied to the nonlinear viscous Burger's equation as an example. Next the method is applied to a three-dimensional aeroelastic model using the CAP-TSD (Computational Aeroelasticity Program Transonic Small Disturbance) code and then to a
\end{abstract}

*Research Engineer

Copyright $c 1997$ by the American Institute of Aeronautics and Astronautics, Inc. No copyright is asserted in the United States under Title 17, U.S. Code. The U.S. Government has a royaltyfree license to exercise all rights under the copyright claimed herein for Governmental purposes. All other rights are reserved by the copyright owner. two-dimensional model using the CFL3D Navier-Stokes code. Comparisons of accuracy and computational cost savings are presented. Because of its mathematical generality, an important attribute of this methodology is that it is applicable to a wide range of nonlinear, discrete-time problems.

\section{INTRODUCTION}

During the early development of mathematical models of unsteady aerodynamic responses, the efficiency and power of superposition of scaled and shifted fundamental responses, or convolution, was quickly recognized. This led to the classical Wagner's function', which is the response of a twodimensional airfoil, in incompressible flow, to a unit step variation in angle of attack. Similar functions such as Kussner's function, which is the response of a two-dimensional airfoil to a sharp-edged gust in incompressible flow, were developed as well'.

As geometric complexity increased, however, the analytical derivation of these time-domain fundamental functions became quite complicated and, therefore, impractical. Ultimately, frequency-domain aerodynamics for threedimensional configurations became the method of choice for computing linear unsteady aerodynamic responses ${ }^{2}$. For the case where geometry-and/or flow-induced nonlinearities are significant in the aerodynamic response, time integration of the nonlinear equations is necessary, as is done in unsteady CFD codes, particularly for aeroelastic analyses. As CFD codes have grown in complexity and capability, there is a very real need to incorporate these codes into aeroservoelastic (ASE) analyses, loads estimation, and other preliminary design efforts in an efficient and accurate manner. Direct incorporation of a CFD code into the ASE process is currently not practical due to the high 
computational costs and turnaround time required. As computational speeds improve and as new algorithms are developed to address this problem, the practicality of this approach may improve. At the moment, however, the efficient incorporation of the information provided by a CFD code into disciplines such as ASE remains a problem.

Attempts to address this problem include the development of transonic indicial responses ${ }^{3.4 .5}$. Reference 6 develops models of nonlinear aerodynamic maneuvers from an experimental database using neural networks. References 7 and 8 provide reduced-order models for linear and linearized solutions about a nonlinear condition.

In order to develop robust, mathematicallycorrect and efficient nonlinear models of the CFD response, a mathematically-formal method is required that is well defined in the time and frequency domains and that is well defined for continuous- and discrete-time systems. The discrete-time Volterra theory of nonlinear systems fulfills these requirements and was applied in the present research. This theory has found wide acclaim in the field of nonlinear discrete-time systems $s^{y}$ and nonlinear digital filters for telecommunications and image processing ${ }^{10}$, to name a small subset of references. Applications of this theory to nonlinear, discretetime aerodynamic systems include Tromp and Jenkins" ${ }^{1}$, Rodriguez ${ }^{12}$, and Silva ${ }^{13.14}$. In Ref. 13, the concept of applying the Volterra theory to the development of efficient linear and nonlinear aerodynamic impulse responses was presented and demonstrated to be feasible for high frequencies. In Ref. 14, the identification, and computational efficiency of linear discrete-time aerodynamic impulse responses, valid for arbitrary inputs, was demonstrated using the linear equations within the CAP-TSD ${ }^{15}$ (Computational Aeroelasticity Program - Transonic Small Disturbance) code. Nonlinear aerodynamic impulse responses were identified using the nonlinear equations within the CAP-TSD code but were limited in scope because of the particular identification technique that was used. The present paper removes these limitations by presenting a mathematicallycorrect identification scheme for nonlinear responses. Reference 14 represents the first time that aerodynamic impulse response functions were numerically identified. The concept of linear and nonlinear aerodynamic impulse response functions introduces a totally new perspective on linear and nonlinear, steady and unsteady aerodynamics, as will be discussed.

The purpose of this paper is to introduce new, or improved, mathematical developments that allow the mathematically-correct identification of linear and nonlinear aerodynamic impulse responses. The functional classification of the discrete-time Navier-Stokes equations that enable the correct application of the discrete-time Volterra theory to CFD codes is presented. The fundamental nature of these responses with regards to classical and modern aerodynamic theories and the impact of these developments on fields such as aeroelasticity and ASE is discussed as well. As an illustrative example, the discretetime Volterra theory is applied to the nonlinear viscous Burger's equation. Then the theory is applied to a three-dimensional aeroelastic model using the CAP-TSD code and then to an airfoil in plunge using the CFL3D ${ }^{16}$ Navier-Stokes code. Comparisons of accuracy and computational cost savings are presented.

\section{MATHEMATICAL PRELIMINARIES}

\section{Discretized Navier-Stokes Equations}

The application of CFD codes involves, in general, the application of the discretized NavierStokes (NS) equations. This is true for the entire spectrum of equation levels, from the linear equations to the full Navier-Stokes equations, including transonic small-disturbance (TSD) and Euler equations. The only difference between the different equation levels is the number and type of simplifying assumptions used to derive the resultant governing equations. It is important, therefore, to understand the functional nature of the NS equations ${ }^{17}$ from a mathematical systems perspective.

Upon convergence of an initial, steady-state solution, the discretized NS equations form a discrete-time, nonlinear, time-invariant system. Reynold's averaging of the NS equations and inclusion of turbulence models to provide closure does not alter this aspect of the equations. This realization, formally stated here for the first time, allows the application of techniques routinely used in the modeling and design of nonlinear, discrete-time filters. In particular, Ref. 18 proves that discrete-time, nonlinear, time-invariant systems with memory can be modeled arbitrarily well using Volterra models, neural networks, or radial basis functions. An important attribute of 
Volterra models is that physical interpretation of the resulting functions is possible, in the time and frequency domains, which often reveals an underlying structure of the system. Description of the Volterra theory of nonlinear systems is presented in Refs. 13 and 14, and the references therein.

A time-invariant system, also referred to as a stationary or autonomous system, is a system whose fundamental properties do not change with time. An example of a simple, time-invariant, nonlinear system is a pendulum. Although the full nonlinear equation of a pendulum is certainly a function of time which exhibits nonlinear, unsteady responses, neither the length of the pendulum nor the mass at the end of the pendulum are functions of time ${ }^{19}$.

A time-varying system, also referred to as a non-stationary or non-autonomous system, is a system whose fundamental properties change with time. Fortunately, for many of the problems in aircraft unsteady aerodynamics, aeroelasticity, and aeroservoelasticity, the governing nonlinear equations are time-invariant. The linearization of these time-invariant, nonlinear equations about an operating point yields the familiar time-invariant, linear equations that comprise the majority of modernday, linear analyses techniques in these fields.

The memory of a system, linear or nonlinear, is a measure of dependence of the system on outputs from previous times. The impulse response of a linear system is the "memory" of that system. It is a temporal representation of the manner in which and the length of time over which a unit perturbation remains active in the response of the system. Convolution then is used to predict the exact response of the linear system to an arbitrary input (any and all steady and unsteady inputs) because all responses of the system are scaled and shifted superpositions of this memory function. Likewise, the concept of memory functions can be extended to nonlinear systems via the Volterra theory of nonlinear systems.

Numerical approximations to ordinary and partial differential equations, such as finitedifference techniques, are defined by the dependence of the response on previous values of input and output. Clearly then, time-accurate, discretized models, such as finite-difference models, are systems with memory, by definition. A discretized version of the NS equations (after steady-state convergence) is, therefore, a time- invariant, nonlinear, discrete-time system and the application of the discrete-time Volterra theory to this system of equations is a valid mathematical approach as proved by Ref. 18 .

\section{Discrete-time Systems}

The modern field of discrete-time signal processing ${ }^{20}$ is a mathematical systems field that addresses substantially more issues than just the sampling of a continuous-time signal. A main topic in this field is that of digital filter design. In digital filter design, there exist mathematical concepts that are quite different from their continuous-time counterparts. The first of these is the unit impulse function, or the Dirac delta function. Whereas the continuous-time unit impulse is an abstract function, typically considered impractical for actual applications ${ }^{21}$ or sometimes misinterpreted as an indicial (step) input $^{6,22.23}$, the discrete-time equivalent, known as the unit sample function, is a simple, welldefined and extremely useful function. Digital filters are designed using this input and its resultant output known as the unit sample response. The unit sample function is defined as

$$
\begin{aligned}
\mathrm{u}[\mathrm{t}] & =1.0 \text { for } \mathrm{k}=\mathrm{k} 0 \\
& =0.0 \text { for } \mathrm{k \neq k} 0
\end{aligned}
$$

The application of this input to a linear, discretetime system will yield the system's unit sample response, the discrete-time equivalent of the unit impulse response. The properties of the unit sample response are identical to those of the unit impulse response. Both responses completely define a linear system and, through convolution, the response of the system to any arbitrary input can be predicted exactly without actually processing the arbitrary input through the system. This is because the unit sample response captures the system's complete frequency content.

A linear system's frequency characteristics can be determined by applying multiple sinusoids of varying frequency, applying band-limited white noise, or by computing the fast Fourier transform (FFT) of the unit sample response. The application of multiple sinusoids is, basically, how linear, frequency-domain, unsteady aerodynamics are generated. The band-limited white noise technique implies exploration of different segments of the system's bandwidth in a 
piecewise, overlapping, and inefficient fashion. The most efficient approach is to compute the FFT of the unit sample response, yielding the system's frequency response. This efficiency is the result of the fundamental properties of the unit sample response. Additional evidence of this efficiency is the fact that the response of the system to the multiple sinusoidal inputs and the band-limited white noise can be computed via convolution of these inputs with the unit sample response. Therefore, from the single computation of the unit sample response, all system responses, from steady (step) to random, can be generated as well. This concept is well understood and routinely applied in the design of digital filters yet appears to be rare in fields dominated by continous-time concepts.

The concept of convolution is another idea that is routinely used in digital filter design but that is perceived as somewhat abstract, and therefore avoided, by the continuous-time community. Because it was believed that practical application of an impulse to an aerodynamic system could not be performed, discrete-time aerodynamic impulse responses were never identified until recently in Ref. 14.

Convolution, in discrete-time, is defined as

$$
y[n]=\sum_{k=0}^{\infty} h[n-k] x[k]
$$

where $h[n-k]$ is the unit sample response and $x[k]$ is the arbitrary input. It is important to understand that this is not the discrete-time version of Duhamel's integral ${ }^{24}$, which is the convolution of a unit step response with the derivative of an arbitrary input. The unit step (indicial) response is not the same as the unit sample (impulse) response, as some references have indicated ${ }^{6.22 .23}$.

The response of a linear system to an arbitrary function of time, $x[k]$, can be computed via three methods. The first, or trivial method, is to process the input through the system itself. If the system is complex and computationally intensive, significant computational costs, including turnaround time, will be incurred. The second method is to identify the system's unit step response and then, via convolution with the derivative of the arbitrary input, obtain the response of the system using $y[n]=x[0] S[n]+\sum_{k=0}^{\infty} S[n-k] x^{\prime}[k]$

where $S[k]$ is the unit step response and $x^{\prime}[k]$ is the derivative of the arbitrary input. Equation (3) is the discrete-time equivalent of Duhamel's integral. The first term in Eq.(3) must, of course, be included whenever $x[0]$ is nonzero. Equation (3) is the correct discrete-time implementation for indicial (or step) aerodynamics. It is mathematically-valid if and only if the step response is correctly identified and applied in Equation (3). The application of step functions has typically been a problem in computational unsteady aerodynamics because of the downwash equation and the perceived problem with the derivative of a step input. This issue is addressed in a subsequent section of this paper.

The third method is to identify the system's unit sample response and, via convolution with the arbitrary input, $x[k]$, (Eq. (2)), obtain the response of the system. Again, proper identification of the unit sample response is a requirement for the succesful application of this method.

Clearly, for complex and computationallyintensive linear systems, the second and third methods provide the most efficient method for computing responses because repeated execution of the system is not required. The unit sample response and the unit step response contain all the necessary information regarding the system's behavior in a compact form. In addition, the derivative of the unit step response is the unit sample response so that only one response, the step or the unit sample response, is needed to compute the other. The derivative of Wagner's function, for example, yields the incompressible, aerodynamic impulse response due to plunge for a two-dimensional airfoil ${ }^{25}$. Figure 1 was obtained using W.P. Jones' approximation to Wagner's function ${ }^{21}$. Details regarding this result and its relation to Theodorsen's function can be found in Ref. 25.

In this research, the identification and use of linear and nonlinear aerodynamic unit sample responses is favored over that of the unit step responses for the following reasons: (1) The unit step response can be computed via convolution of the unit sample response with a step input, yielding the steady-state solution; and (2) 
Convolution using the unit sample response involves the actual input whereas convolution using the unit step response involves the derivative of the input, requiring additional, unnecessary computational effort. The unit sample response is the most compact representation of a linear system from which all other steady and unsteady responses can be generated. Extension of this concept to nonlinear systems then enables the efficient computation of nonlinear steady and unsteady responses due to arbitrary inputs.

Identification of linear aerodynamic unit sample responses ${ }^{14}$ has interesting implications. First, it provides an alternative to the forced harmonic method for computing unsteady aerodynamic forces by computing the unit sample responses for each mode and then performing the convolutions with sinusoidal inputs of varying frequency. This could be done more directly by performing a Fourier transform of each of the modal unit sample responses.

The unsteady aerodynamic frequency domain may be avoided altogether by performing the aeroelastic analyses directly in the time domain ${ }^{13}$. This is done by coupling the aerodynamic unit sample responses with the linear structure in a closed-loop sense and obtaining the time-accurate aeroelastic transients. Since the aerodynamic unit sample response is valid in the complex plane, there is no need for rational function approximations ${ }^{23}$ (RFAs) that extend the forcedharmonic responses, valid only along the imaginary axis, to the complex plane via analytic continuation. Current methods for generating RFAs, limited by a specified frequency range of interest to generate a low-order model, are actually modeling that portion of the unit sample response that contains the particular frequency range of interest ${ }^{13}$. The aerodynamic unit sample response can also be used to realize a linear, discrete-time, state-space system ${ }^{26}$. This approach was investigated by the author and will be the subject of another paper.

Linear frequency domain and RFA methods are not applicable to nonlinear aerodynamics and, consequently, the generation of time-accurate, aeroelastic transients is necessary. The discretetime Volterra theory of nonlinear systems, along with new mathematical developments presented in this paper, provides a formal method for the identification of nonlinear unit sample responses. This results in significant computational efficiency when applied, for example, to a CFD code.

\section{Aerodynamic System Input Definition}

An important conceptual development of Ref. 14, and its subsequent improvement in the present research, was the mathematically-correct definition of the input to an unsteady aerodynamic system for the discrete-time domain. The input function consists of the downwash function, which, for the excitation of a given mode is written as

$$
w(x, y, t)=p h i^{\prime}(x, y) \bullet u(t)+p h i(x, y) \bullet u^{\prime}(t)
$$

where $\operatorname{phi}(x, y)$ is the modeshape, phi' $(x, y)$ are the slopes of the modeshape, $u(t)$ is the generalized coordinate, and $u^{\prime}(t)$ is the derivative of the generalized coordinate. The discussion will be limited, temporarily, to the linear case.

The current method for the excitation of aeroelastic modes within a CFD code involves the definition of a "smooth" function defined as

$$
u(t)=d_{0} * \exp \left(-w\left(t-t_{0}\right) * * 2\right)
$$

where $d_{0}$ is the maximum amplitude desired, $w$ is the width, and $t_{0}$ is the time at which the maximum amplitude is reached. This Gaussian curve (Equation (5)) is referred to as the exponential pulse function. This exponential pulse is input to each of the modes of the system to obtain the set of exponential pulse responses, about a nonlinear steady state solution ${ }^{27.28}$ that are then transformed to the frequency domain for use in standard linear analyses techniques. This should not be confused with the unit pulse response mentioned throughout this paper. Whereas the unit pulse input (Eq. (1)) excites all the frequencies for a given mode, the exponential pulse input will excite only the particular range of frequencies defined by the width of the exponential pulse. This can be explained using Eq. (4) as follows.

From Equation (5), the downwash equation consists of the first term which multiplies $u(t)$ by the slopes of the modeshape added to $u^{\prime}(t)$ multiplied by the modeshape. When the shape of $u(t)$ is narrowed, then the derivative term, $u^{\prime}(t)$, is much bigger and changes more rapidly than it 
does for the wider pulse, thereby exciting higher frequencies. Shape optimization may, therefore, have to be performed to obtain the desired frequency range of interest. Typically, a "wide" pulse is recommended, forcing the $u$ ' $(\mathrm{t})$ term to be small.

A critical drawback, however, is that the exponential pulse is perceived, erronously, as a single input. That is, the fast Fourier Transform (FFT) of the output generalized force is divided by the FFT of the perceived single input, $u(t)$, to obtain the linearized frequency response function for that generalized force. But inspection of Eq. (4) clearly shows that the downwash function is a two-input function. The user defines $u(t)$ but the quantity that is input to the flow solver is Eq. (4), which includes the effect of $u^{\prime}(t)$ as well. Because this derivative is computed analytically internal to the code, it is invisible to the user, although it is certainly not invisible to the flow solver.

Inspection of Eq. (4) for a plunge mode reveals that the first term is identically zero because the slopes of a plunge mode are zero. Therefore, the only temporal function that is actually input to the flow solver is $u^{\prime}(t)$. For a plunge mode, the denominator of the frequency response function should be the FFT of $u^{\prime}(t)$, not the FFT of $u(t)$. This will be demonstrated using convolution with examples from CAP-TSD and CFL3D in the results section of this paper.

It is because this second term of the downwash input has been ignored that wiggles appear at lower or higher frequencies, depending on the input $u(t)$, in early applications of the technique $^{27}$. The reason for the success of the technique to date is that for most modes, a very wide $u(t)$ term results in a very small $u^{\prime}(t)$ term, thereby exciting, predominantly, the lower frequency range which is, typically, where most analyses are desired anyway. If an accurate determination of the entire frequency range of a mode is desired, then the second term of the downwash function must be included in the analysis. In terms of computational efficiency, the exponential pulse response does not possess any of the mathematical properties of the unit sample response nor can it be formally extended to nonlinear systems.

The misinterpretation of the downwash as a single input has led to the false conclusion that impulse (or unit pulse) and step inputs cannot be applied to a CFD code because these inputs will result in numerical difficulties. The reasoning is that the application of a unit pulse, or unit step, input as $u(t)$ would lead to a very large, if not infinite, derivative term, $u^{\prime}(t)$. So typically, a step input is modified, or made "smoother", so that the $u$ '( $t$ ) does not cause numerical problems. These "smoother" responses, however, are not mathematically consistent with the strict definition of unit pulses or unit step inputs and so will yield inaccuracies when used in convolution. The unit pulse and unit step functions have a very precise mathematical description which allows for convolution to be applied. Any deviation from this precise definition will reduce, or possibly eliminate, the accuracy of the convolution.

Mathematically, the downwash equation (for a given mode) is clearly a two-channel input. For the linear case, each term of the downwash equation can, and should, be treated as a separate input channel' ${ }^{14}$. For the nonlinear case, the response due to the sum of the terms of the downwash will not be equal to the sum of the separate responses due to each term of the downwash. The inputs, however, still need to be treated as independent inputs. This difficulty was solved by computing a combined unit sample response that consists of a unit sample input applied to each of the two inputs simultaneously while using a deconvolution ${ }^{25}$ technique to maintain mathematical accuracy. This deconvolution technique identified the proper temporal function that can be used with the combined unit sample response to yield the correct final response for the linear case. Since the combined motion of the system due to the combined inputs of the downwash is the same for the linear and nonlinear cases, the same combined motion is used in the linear and nonlinear convolutions. The effectiveness of this method will be presented in the results section of this paper.

\section{VOLTERRA THEORY}

The discrete-time Volterra series for a truncated, second-order, time-invariant, system has the form 


$$
\begin{aligned}
y[n]= & h_{0}+\sum_{k=0}^{N} h_{1}[n-k] x[k]+ \\
& \sum_{k 1=0}^{N} \sum_{k 2=0}^{N} h_{2}[n-k 1, n-k 2] x[k 1] \times[k 2]
\end{aligned}
$$

where $y[n]$ is the response of the nonlinear system to $x[k]$, an arbitrary input; $h_{0}$ is the mean value about which the response is defined; $h_{1}$ is the first-order kernel or the linear unit sample response; and $h_{2}$ is the second-order kernel. Details of the theoretical definitions of this method, including identification of the kernels, can be found in Refs. 13, 14 and all the references therein. As in Refs. 13 and 14, modeling of the nonlinear aerodynamic system will be limited to identification of the first- and second-order kernels.

An intuitive explanation of the application of this approach to a nonlinear system can be stated as follows. It is a well-established procedure to linearize a nonlinear system by expanding the nonlinear terms in a Taylor Series about a chosen point. The resultant Taylor Series, if expanded to sufficient terms, is an excellent approximation to the actual nonlinearity. That is, there are no restrictions on its range of applicability regarding input amplitudes. As the series is truncated by gradual elimination of the higher-order terms from highest to lowest, limitations on the range of applicability of the series approximation become more restrictive until the only term left is the linear term, the most severely restricted term of all. If higher-order terms are gradually added back in to the series approximation, one at a time, the accuracy of the approximation is improved and the range of applicability is increased as well. The present method is, therefore, a method that re-instates higher-order terms that were removed during the linearization of the equations. This will yield improved accuracy over the purely linear solution and will increase the range of applicability as well.

Also, when a "small" (or "linear") input is applied to a nonlinear system, there is an implicit assumption of the equivalence between the nonlinearity and its series expansion. This is evident because it is in the presence of a series expansion formulation that a "small" input will, in fact, yield the "linear" portion of the response since the higher-order terms (second-order and above) are much smaller and, therefore, negligible. The accepted practice of using a "small" amplitude exponential pulse response (within a CFD code, for example) to excite only the "linear" portion of the response about a nonlinear solution implies a series approximation to the nonlinearity. As a result, this "small" input approach offers additional validation to the present application of the discrete-time Volterra theory, which seeks to identify the next higher-order term after the linear term.

Furthermore, the first-order term is more accurate than the purely linear term because the first-order term is derived with knowledge of the second-order, or higher-order, terms. Therefore, for a second-order nonlinearity, the first-order term is the proper and correct linearization. The first-order term can be considered to represent a "mean" value of the response with the secondorder term representing a higher-order variation about that mean.

The success of linearized aerodynamic predictions for certain flight regimes, and under certain small perturbation assumptions, is due to the fact that highly nonlinear phenomena have a negligible impact on the net effect of various responses at these conditions. It does not mean that rotational, viscous, and turbulent effects disappear from the flow at these conditions, but rather that these effects do not excite higher-order effects sufficiently to affect the overall response. Increasing the order of this restricted linearized approximation to model higher-order effects is, therefore, a logical step.

The computational efficiency of the present technique is due to the following features of the method. 1). Identification of the first- and second-order kernels eliminates the need to reexecute the code. 2). The kernels can be coupled with a structure in a closed-loop sense "outside" of the CFD code, on a workstation, sidestepping the current, very expensive method of solving the aeroelastic equations of motion within the CFD code. 3). The identification of the kernels is geometry independent. The kernel of a threedimensional configuration is, topologically, no different from the kernel of a two-dimensional configuration. The only difference is the initial cost of identification that requires the use of the CFD code. The complex CFD model, consisting of three spatial variables and one temporal variable, is mapped onto the unit sample 
response, a compact function of time only. The modal approach and the definition of boundary conditions within a CFD code make this mapping possible. 4). This technique permits a unified approach for generation of compact, linearized and nonlinear, steady and unsteady models from the same, arbitrarily complex CFD model (complete configuration, finest grid, most detail), including, of course, stability derivatives.

\section{RESULTS}

\section{Linear CAP-TSD}

The linear equations within the CAP-TSD code were used for comparisons of unit sample and step responses. The computational model is a rectangular wing with an aspect ratio of two. All results presented are for $M=0.9$. Shown in Figure 2 is a comparison of the plunge unit sample response and the plunge unit step response. Convolution of the unit sample response with a unit step also yields the unit step response, as shown in Figure 3. Convolution of the unit sample response with the input shown in Figure 4, $\mathrm{u}^{\prime}(\mathrm{t})$, yields the exact, CAP-TSDgenerated result, also shown in Figure 4. Convolution of the plunge unit sample response with $u^{\prime}(t)$, instead of $u(t)$, yields the correct result, consistent with the discussion regarding Equation (4) in a previous section.

These results demonstrate the relationship between a unit sample response and a unit step response for a linear unsteady aerodynamic system and the correct application of these functions. Also, it is important to realize that the unit sample response, when convoluted with a step input results in the steady-state solution, as shown. Therefore, unit sample responses can be used for predicting the steady and unsteady responses of a system. This applies to the nonlinear case as well where the savings in computational cost and time are of greater importance.

\section{Viscous Burger's Equation}

The 1-D viscous Burger's equation is defined as

$$
\frac{\partial u}{\partial t}+u \frac{\partial u}{\partial x}=v \frac{\partial^{2} u}{\partial x^{2}}
$$

and is typically used as a simplified model of the Navier-Stokes equations for evaluating the effectiveness of numerical methods ${ }^{29}$. It is used here to demonstrate the effectiveness of the discrete-time Volterra technique. The numerical solution is implemented via a simple forward-intime, central-in-space (FTCS) method.

The identification part of the process consists of the generation of the first- and second-order kernels of a selected grid point due to perturbation of the end-point boundary condition. Shown in Figure 5 is the first-order kernel of the system, revealing a well-behaved and compact function. Shown in Figure 6 are the first twenty terms of the symmetric second-order kernel. These terms indicate a second-order nonlinear memory that goes to zero fairly quickly.

Shown in Figure 7 is a comparison of several responses due to step inputs of increasing amplitude for the actual numerical solution, the convolution of the first-order kernel with each of the inputs, and the convolution of first- and second-order kernels with each of the inputs. As the amplitude is increased, the error between the actual ("true") response and the first-order response increases, indicating an increasing effect of the nonlinearity as amplitude is increased. Addition of the second-order convolution shows a significant improvement in accuracy, as seen in Figure 7. The crossing over of the convolved response for the largest step response could be an indication of a convergence limit or the need for additional terms of the second-order kernel. The improvement in response with the addition of the second-order term is, nontheless, evident. Using only the first-and second-order kernels, steadystate responses of the nonlinear system can be computed without re-execution of the actual numerical system. It is interesting to note that, for a certain range of amplitudes, the first-order response may be sufficient, depending on the level of accuracy desired.

Actual and convolved responses, using the same first- and second-order kernels, due to sinusoidal inputs were generated ${ }^{25}$. Shown in Figure 8 is the comparison for one of these inputs. Again, the comparisons were excellent with the combined first- and second-order response showing the best agreement with the actual responses. In the case of a purely linear system, these responses could be used to generate the frequency response function of the system, as is done in the doublet lattice technique for linear aerodynamic systems. Therefore, whereas the unit sample responses are valid in the complex domain, the forced harmonic response, which can 
be generated from the unit sample response, is valid only on the imaginary axis. The unit sample responses (linear) and first- and secondorder kernels (nonlinear) do not have any such limitation. The only limitation of the nonlinear functions is that the radius of convergence of the series is limited by the norm of the input ${ }^{13,14}$, which depends on the system being investigated. These functions are therefore more powerful and, at the same time, more efficient than any other responses that can be obtained from a given system. This is because all other system responses are the result of a convolution of the system's unit sample response with some arbitrary input.

Shown in Figure 9 is a comparison of the actual, first-order, and first- plus second-order responses due to a quasi-random input from a uniform probability distribution. Again, the comparisons are reasonable for the first-order only and excellent for the first-plus second-order responses. This is analogous to the computation of the response of a nonlinear system (aircraft) due to a random input, such as a gust. Therefore, just as in the linear case, the first-and secondorder kernels can be used to predict the response of the nonlinear system to any arbitrary input, which is an infinite set of possible inputs.

\section{Nonlinear CAP-TSD}

The nonlinear TSD equation is solved within the CAP-TSD code for a rectangular wing with a NACA0012 airfoil section undergoing plunge and an aspect ratio of two at a Mach number of 0.9 . Figure 10 is a comparison of nonlinear CAP-TSD responses, due to plunging motions of different amplitudes, with the convolved results of the first-order kernel with the same inputs. The linear CAP-TSD result for the first amplitude is also shown for comparison. For this mode, the first-order kernel seems to be sufficient to capture the range of responses. This is not surprising given the nature of the TSD equation. The cost for ten of these types of responses using CAP-TSD directly is 38,000 CPU secs and 15 hours turnaround time. The cost using the first-order convolution for ten of these types of responses is 4,150 CPU secs and 2.04 hours turnaround time. Most of the cost of the first-order convolution is the initial identification part of the process since each convolution itself took only 75 seconds on a workstation. As the need for the response of the system to arbitrary inputs (motions) increases, the cost of the method decreases because once the unit sample responses are obtained, the CFD code need not be re-executed.

Figure 11 is a comparison of the actual nonlinear CAP-TSD solution for the same wing undergoing an arbitrary pitching motion and the response obtained by the convolution of the combined first-order kernel and the appropriate input, obtained as described in an earlier section of the paper. The comparison is reasonable, but for this mode, the second-order terms are needed ${ }^{25}$. The computational efficiency has, however, been doubled and is now mathematically correct for nonlinear responses. The reason for this is that instead of computing two responses per mode (one for each term of the downwash function, Eq. (4)), only one response per mode is needed.

\section{CFL3D (version 5.0)}

Navier-Stokes results for a dense-grid RAE airfoil $^{16}$ with the Spalart-Allmaras turbulence model undergoing plunge at $\mathrm{M}=0.75$ were computed at a time step of 0.001 . The RAE airfoil grid is presented in Figure 12.

Comparison of the CFL3D responses with the first-order convolved responses, as well as a linear response, are shown in Figure 13. The comparisons are excellent, with decreasing accuracy as the amplitude increases, similar to the viscous Burger's equation results. As amplitude increases, so does the need for secondorder kernels. Details for this case and higher Mach numbers (increased nonlinearity) can be found in Ref. 25.

These results prove the applicability of discrete-time, nonlinear, unit sample responses at the NS equation level, as discussed in the beginning of the paper.

The cost of each CFL3D run was about 2,000 CPU seconds. The cost of the first-order kernel identification was $400 \mathrm{CPU}$ seconds because the kernel goes to zero in less than 100 time steps. The cost of each convolution, performed on a workstation, was 30 seconds. The most important point, however, is that a compact model has been identified that is valid for a range of amplitudes without re-execution of the code.

\section{CONCLUSIONS}

The mathematically correct and numericallyaccurate identification of linear and nonlinear, 
discrete-time aerodynamic impulse responses was presented. For the linear case, the aerodynamic impulse response functions were used to reproduce exactly the responses of a linearized three-dimensional aeroelastic CFD model to arbitrary aeroelastic input motions at a fraction of the computational cost and time. It was shown that the response to step (steady), sinusoidal, and random inputs can all be computed from an impulse response function, establishing the aerodynamic impulse response function as the most fundamental aerodynamic function that can be extracted from a discrete-time, aerodynamic system.

For the nonlinear case, the existence, identification, and application of nonlinear, discrete-time, aerodynamic impulse responses was presented. Applications of the method to the nonlinear viscous Burger's equation revealed the existence of well-behaved first- and second-order impulse response functions. The method was then applied to nonlinear aeroelastic CFD models using the CAP-TSD and CFL3D codes. The results prove the existence of these functions for complex, three-dimensional CFD models and their application demonstrates their accuracy and computational efficiency.

\section{REFERENCES}

'Jones, Robert T., "The Unsteady Lift of a Wing of Finite Aspect Ratio," NACA Report No. $681,1940$.

${ }^{2}$ Giesing, J.P.; Kalman, T.P.; and Rodden, W.P., "Subsonic Unsteady Aerodynamics for General Configurations, Part I. Direct Application of the Nonplanar Doublet Lattice Method. Report AFFDL-TR-71-5, Vol. I, November 1971.

${ }^{3}$ Ballhaus, W. F.; and Goorjian, P. M., "Computation of Unsteady Transonic Flows by the Indicial Method," AIAA Journal, Volume 16, No. 2, February 1978, pp. 117-124.

${ }^{4}$ Leishman, J.; and Crouse, G., "A StateSpace Model of Unsteady Aerodynamics in a Compressible Flow for Flutter Analyses," AlAA Paper No. 89-0022, 27th Aerospace Sciences Meeting, January 9-12, 1989, Reno, Nevada.
${ }^{5}$ Nixon, D., "Alternative Methods for Modeling Unsteady Transonic Flows, Unsteady Transonic Aerodynamics, Vol. 120 of Progress in Astronautics and Aeronautics, Ed. by D. Nixon, AIAA, 1989.

${ }^{6}$ Riesenthal, P.H., "Development of a Nonlinear Indicial Model for Maneuvering Fighter Aircraft," AIAA Paper No. 96-0896, 34th Aerospace Sciences Meeting \& Exhibit, January 15-18, 1996, Reno, Nevada.

'Dowell, E.H., "Eigenmode Analysis in Unsteady Aerodynamics: Reduced Order Models," 36th AIAA/ASME/ASCE/AHS/ASC Structures, Structural Dynamics, and Materials Conference, New Orleans, 1995.

${ }^{8}$ Baker, M.L., "Model Reduction of Large, Sparse, Discrete Time Systems with Application to Unsteady Aerodynamics," PhD Dissertation, University of California at Los Angeles, 1996.

${ }^{9}$ Diaz, Hernando, "Modeling of Nonlinear Systems from Input - Output Data," PhD Dissertation, Rensselaer Polytechnic Institute, 1986.

I"Pitas, I.; and Venetsanopoulos, A.N., Nonlinear Digital Filters: Principles and Applications, Kluwer Academic Publishers, 1990.

"Tromp, J. C.; and Jenkins, J. E.: "A Volterra Kernel Identification Scheme Applied to Aerodynamic Reactions," AIAA Paper No. 902803, Presented at the AIAA Atmospheric Flight Mechanics Conference, Portland, Oregon, August 20-22, 1990.

${ }^{12}$ Rodriguez, E.A., "Linear and Nonlinear Discrete-Time State-Space Modeling of Dynamic Systems for Control Applications," PhD Dissertation, Purdue University, December 1993.

${ }^{13}$ Silva, W.A., "Application of Nonlinear Systems Theory to Transonic Unsteady Aerodynamic Responses," Joumal of Aircraft, Volume 30, Number 5, September-October 1993, pp. 660-668.

${ }^{14}$ Silva, W.A., "Extension of a Nonlinear Systems Theory to General-Frequency Unsteady 
Transonic Aerodynamic Responses," AIAA Paper No. 93-1590, Presented at the 34th Structures, Structural Dynamics, and Materials Conference, La Jolla, California, April 19-21, 1993.

${ }^{15}$ Batina, J.T., "Unsteady Transonic Algorithm Improvements for Realistic Aircraft Configurations," Joumal of Aircrafi, Vol. 26, Feb. 1989, pp. 131-139.

${ }^{16}$ Krist, S.L.; Biedron, R.T.; and Rumsey, C.L., "CFL3D User's Manual (Version 5.0)," November 1996, First Edition.

${ }^{17}$ Temam, R., Navier-Stokes Equations and Functional Analysis, CBMS-NSF Regional Conference Series in Applied Mathematics, Society for Industrial and Applied Mathematics, Philadelphia, Pennsylvania, 1995.

${ }^{18}$ Park, J.; and Sandberg, I.W., "Criteria for the Approximation of Nonlinear Systems," IEEE Transactions on Circuits and Systems I: Fundamental Theory and Applications, Volume 39, No. 8, August 1992, pp673-676.

${ }^{19}$ Khalil, H.K., Nonlinear Systems, Macmillan Publishing Company, New York, 1992.

${ }^{211}$ Oppenheim, A.V.; and Schafer, R.W., Discrete-Time Signal Processing, Prentice Hall Signal Processing Series, A. V. Oppenheim, series editor, Englewood Cliffs, NJ, 1989.

${ }^{21}$ Fung, Y.C., An Introduction to the Theory of Aeroelasticity, Dover Publications, Inc., New York, 1969.

${ }^{22}$ Dowell, E.H., et al, A Modern Course in Aeroelasticity, Sijthoff \& Noordhoff, The Netherlands, 1980.

${ }^{2.3}$ Edwards, J.W., "Applications of Laplace Transform Methods to Airfoil Motion and Stability Calculations," AIAA/ASME/ASCE/ AHS 20th Structures, Structural Dynamics, and Materials Conference, April 4-6, 1979, St. Louis, MO., pp465-481.

${ }^{24}$ Wylie, C.R., Jr., Advanced Engineering Mathematics, Second Edition, McGraw-Hill Book Company, Inc., 1960.
${ }^{25}$ Silva, W.A., "Identification of Linear and Nonlinear Aerodynamic Impulse Responses Using Digital Filter Techniques," PhD Dissertation, to be published Fall 1997.

${ }^{26}$ Juang, J.-N. ; and Pappa, R.S., "An Eigensystem Realization Algorithm for Modal Parameter Identification and Model Reduction," Joumal of Guidance. Control, and Dynamics, Vol. 8, No. 5, September-October 1985, pp.620627.

${ }^{27}$ Seidel, D.A.; Bennett, R.M.; and Ricketts, R.H., "Some Recent Applications of XTRAN3S," AIAA Paper No. 83-1811, July 1983.

${ }^{28}$ Lee-Rausch, E. M., and Batina, J. T.: "Wing Flutter Computations Using an Aerodynamic Model Based on the Navier-Stokes Equations," Journal of Aircraft, Vol. 33, no. 6, pp.1139-1148, Nov-Dec 1996. (Also AIAA Paper 93-3476, Aug. 1993).

${ }^{29}$ Anderson, D.A.; Tannehill, J.C.; and Pletcher, R.H., Computational Fluid Mechanics and Heat Transfer, Series in computational methods in mechanics and thermal sciences, Hemisphere Publishing Corporation, McGrawHill Book Company, 1984. 


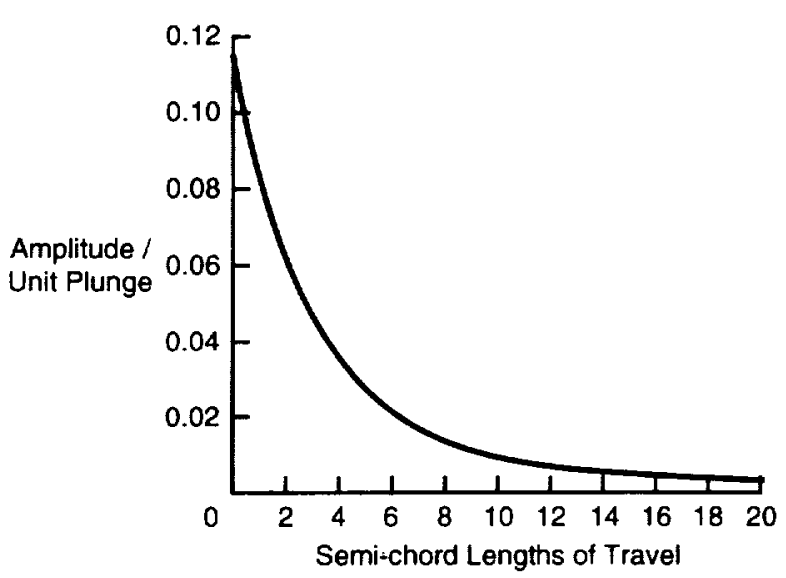

Figure 1 Impulse response due to unit plunge, derived from W.P. Jones' approximation to Wagner's function.

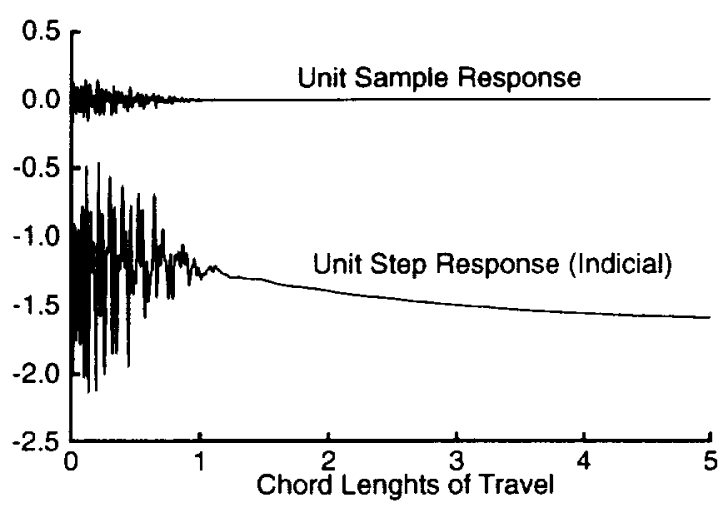

Figure 2 Comparison of unit sample and indicial responses linear, $\mathrm{M}=0.9, \mathrm{DT}=0.001$

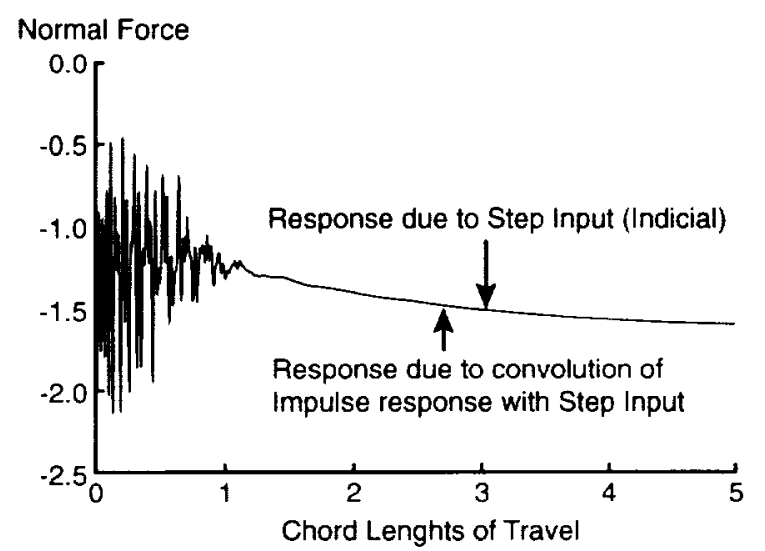

Figure 3 Comparison of indicial responses, linear. $\mathrm{M}=0.9, \mathrm{DT}=0.001$

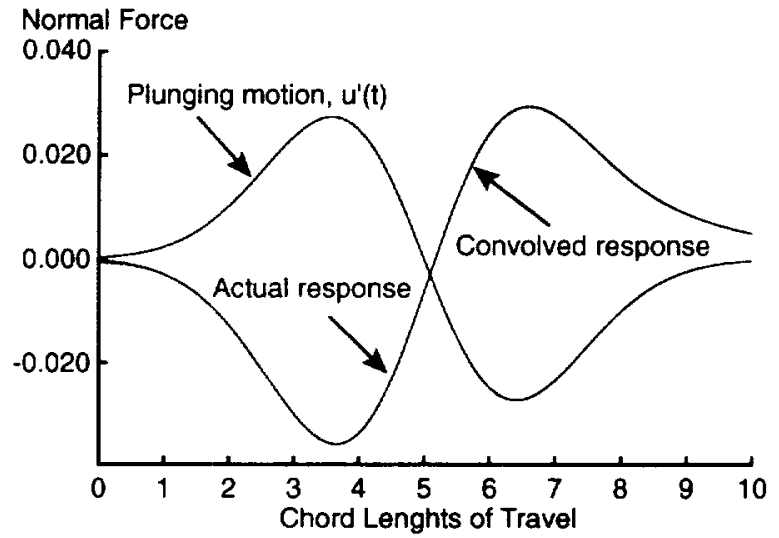

Figure 4 Comparison of responses, due to arbitrary plunging motion, linear, $\mathrm{M}=0.9, \mathrm{DT}=0.001$

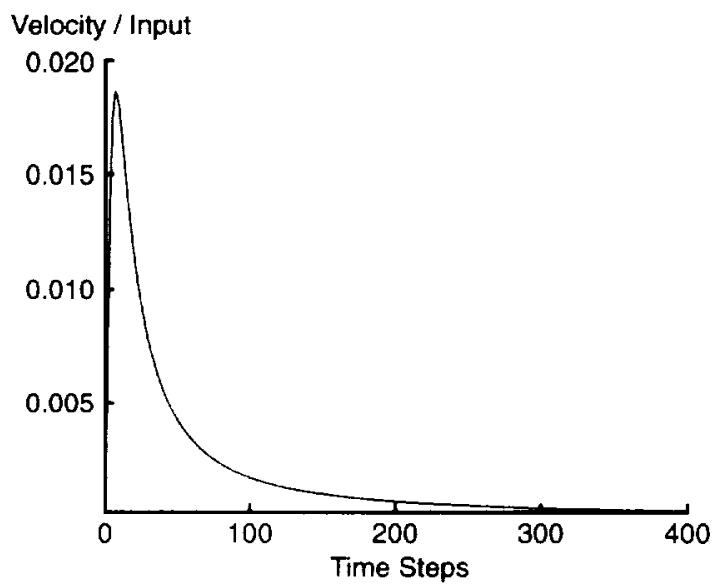

Figure 5 The first-order kernel of the response in velocity to unit perturbation for the viscous Burger's equation.

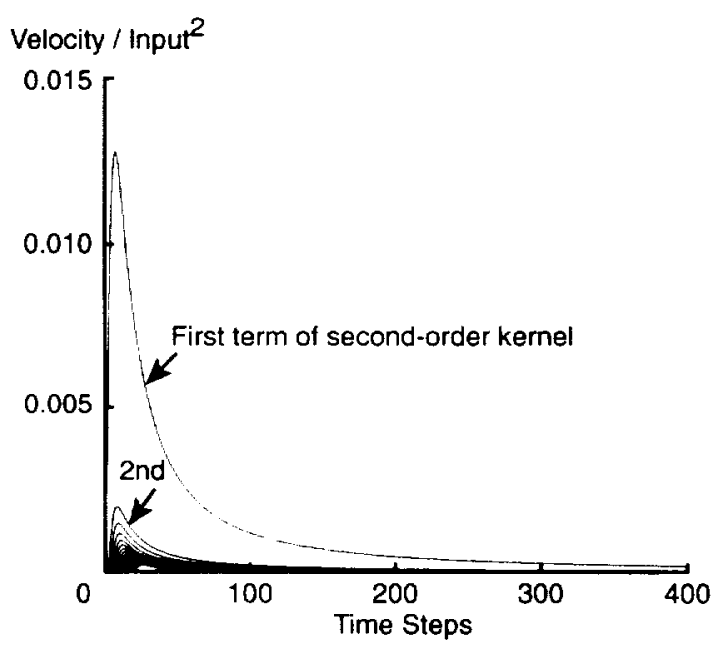

Figure 6 Twenty terms of the second-order kernel of velocity due to unit perturbation squared for the viscous Burger's equation. 


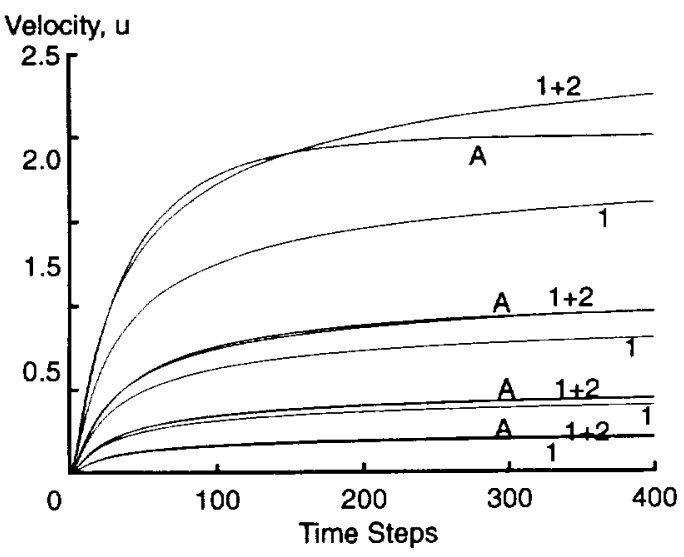

Figure 7 Comparison of actual (A), first-order (1), and first-+ second-order $(1+2)$ step responses for the viscous Burger's equation.

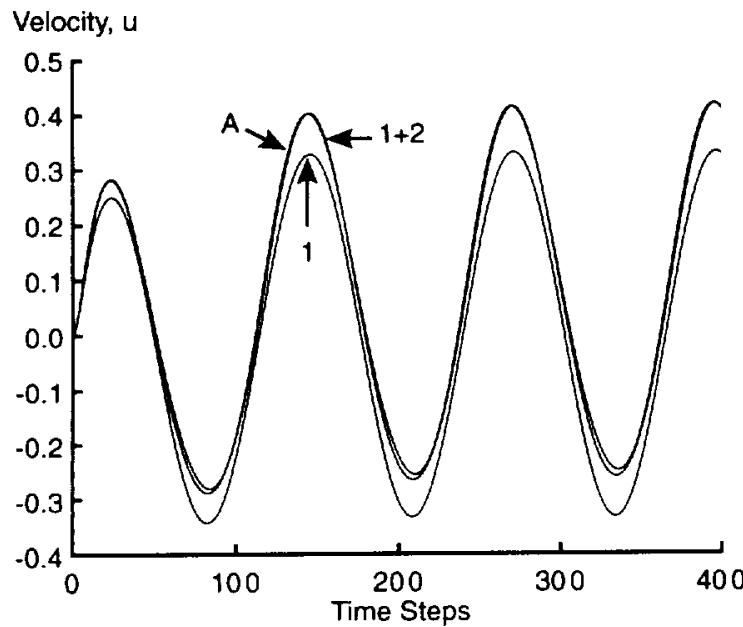

Figure 8 Comparison of actual (A), first-order (1), and first-+ second-order $(1+2)$ harmonic responses for the viscous Burger's equation.

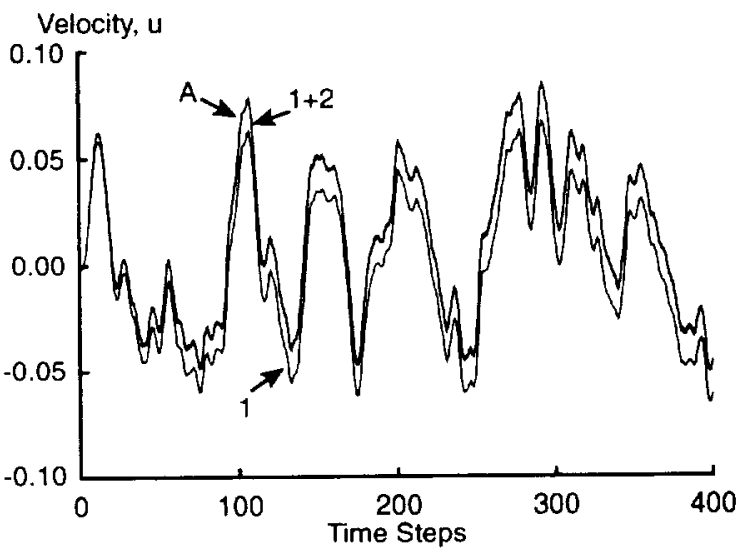

Figure 9 Comparison of actual (A), first-order (1), and first- + second-order $(1+2)$ responses due to quasirandom imput for the viscous Burger's equation.

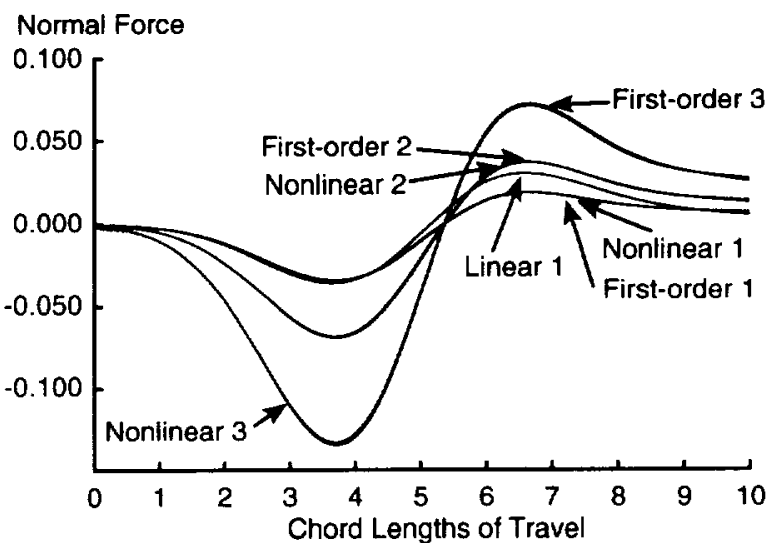

Figure 10 Comparison of actual nonlinear and first-order convolved for three plunging motions, $\mathrm{M}=0.9, \mathrm{DT}=0.001$

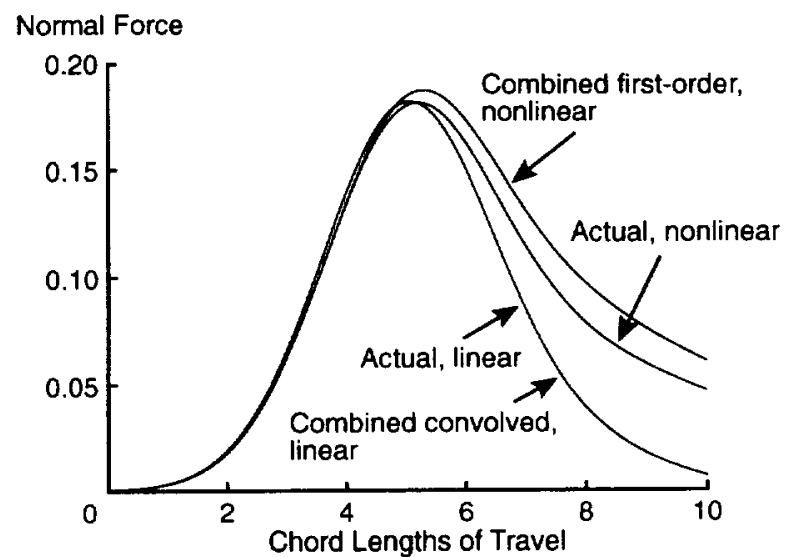

Figure 11 Comparison of linear and nonlinear, actual and convolved, responses for CAP-TSD model pitching at $\mathrm{M}=0.9$.

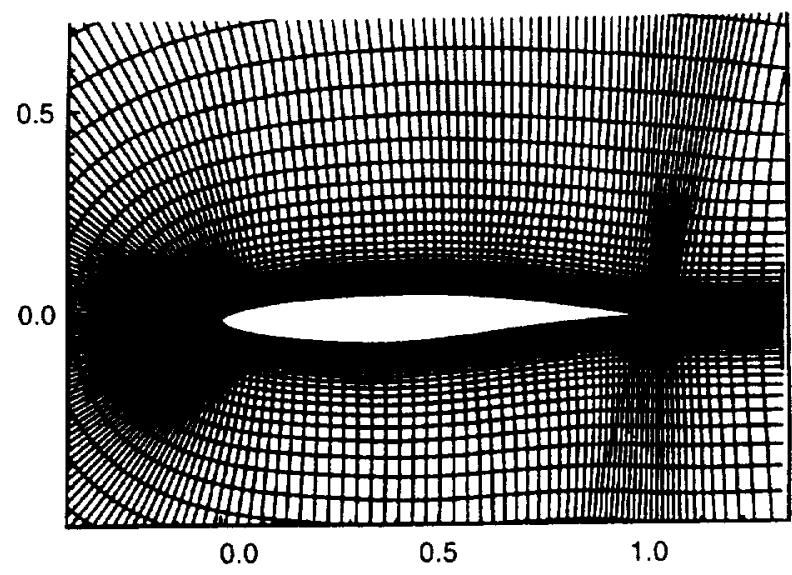

Figure 12 RAE 2822 airfoil grid, from Ref. 16. 


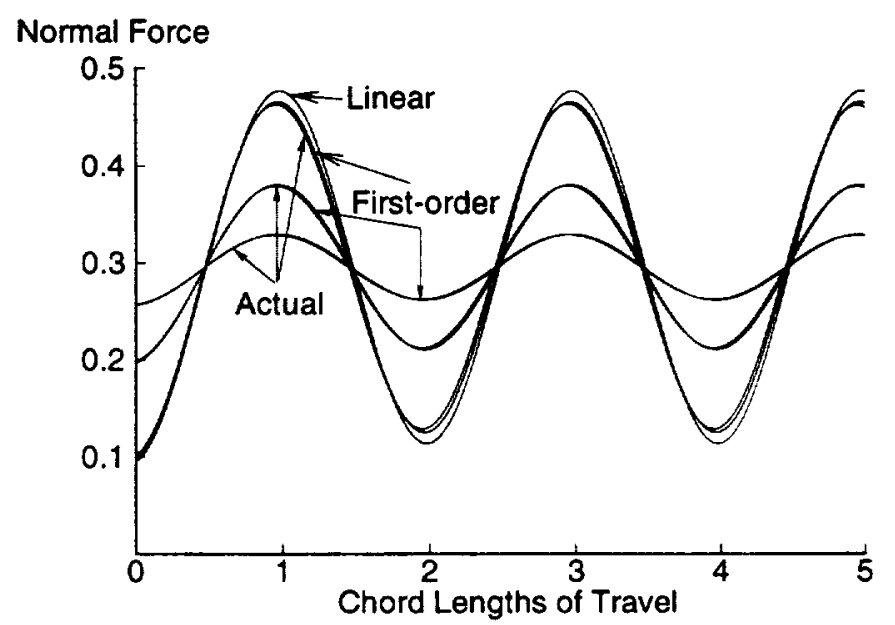

Figure 13 Comparison of plunge responses for RAE airfoil at $M=0.75$, Navier-Stokes with $S-A$ turbulence model. 